\title{
Short-term treatment with GLP-1 increases pulsatile insulin secretion in Type II diabetes with no effect on orderliness
}

\author{
C. B. Juhl ${ }^{1}$, O. Schmitz ${ }^{1}$, S. Pincus ${ }^{2}$, J.J. Holst ${ }^{3}$, J. Veldhuis ${ }^{4}$, N. Pørksen ${ }^{1}$ \\ ${ }^{1}$ Department of Medicine M (Endocrinology and Diabetes), University Hospital of Aarhus, Aarhus, Denmark \\ ${ }^{2}$ Guilford, Connecticut, USA \\ ${ }^{3}$ Department of Medical Physiology, University of Copenhagen, Denmark \\ ${ }^{4}$ Endocrinology Division, General Clinical Research Center, University of Virginia, Charlottesville, Virginia, USA
}

\section{Abstract}

Aims/hypothesis. The enteric incretin hormone, glucagon-like peptide-1 (GLP-1), is a potent insulin secretagogue in healthy humans and patients with Type II (non-insulin-dependent) diabetes mellitus. In this study we assessed the impact of short-term GLP-1 infusion on pulsatile insulin secretion in Type II diabetic patients.

Methods. Type II diabetic patients $(n=8)$ were studied in a randomised cross-over design. Plasma insulin concentration time series were obtained during basal conditions and during infusion with saline or GLP-1 $\left(1.2 \mathrm{pmol} / 1 \cdot \mathrm{kg}^{-1} \cdot \mathrm{min}^{-1}\right)$ on 2 separate days. Plasma glucose was clamped at the initial concentration by a variable glucose infusion. Serum insulin concentration time series were evaluated by deconvolution analysis, autocorrelation analysis, spectral analysis and approximate entropy.

Results. Serum insulin concentrations increased by approximately $100 \%$ during GLP-1 infusion. Pulsatile insulin secretion was increased as measured by secretory burst mass $(19.3 \pm 3.8$ vs $53.0 \pm 10.7 \mathrm{pmol} / \mathrm{l} /$ pulse, $p=0.02$ ) and secretory burst amplitude $(7.7 \pm 1.5 \mathrm{vs} 21.1 \pm 4.3 \mathrm{pmol} / \mathrm{l} / \mathrm{min}, p=0.02)$. A similar increase in basal insulin secretion was observed $(3.6 \pm 0.9$ vs $10.2 \pm 2.2 \mathrm{pmol} / \mathrm{l} / \mathrm{min}, p=0.004)$ with no changes in the fraction of insulin delivered in pulses $(0.50 \pm 0.06$ vs $0.49 \pm 0.02, p=0.84)$. Regularity of secretion was unchanged as measured by spectral analysis (normalised spectral power: $5.9 \pm 0.6$ vs $6.3 \pm 0.8$, $p=0.86$ ), autocorrelation analysis (autocorrelation coefficient: $0.19 \pm 0.04$ vs $0.18 \pm 0.05, p=0.66)$ and the approximate entropy statistic $(1.48 \pm 0.02$ vs $1.51 \pm 0.02, p=0.86)$.

Conclusion/interpretation. Short-term stimulation with GLP-1 jointly increases pulsatile and basal insulin secretion, maintaining but not improving system regularity in Type II diabetic patients. [Diabetologia (2000) 43: 583-588]

Keywords GLP-1, Insulin, pulsatility, insulin secretion, time series, Type II diabetes, human.
Type II (non-insulin-dependent) diabetes mellitus is among a vast number of abnormalities characterised by disturbed beta-cell function. This includes defects in first-phase and second-phase insulin secretion as

Received: 11 November 1999 and in revised form: 13 December 1999

Corresponding author: C. B. Juhl, Department of Medicine M (Endocrinology and Diabetes), Aarhus Kommunehospital, Noerrebrogade 44, 8000 Aarhus, Denmark Abbreviations: GLP-1, Glucagon-like peptide-1; ApEn, approximate entropy. well as a deterioration of the highly coordinated pulsatile release pattern seen in healthy subjects $[1,2]$. The full impact on glucose control of the latter alterations in Type II diabetic patients is not established, but in vivo experiments suggest that pulsatile insulin delivery is important for insulin actions [3-5].

Stimulation of insulin secretion has been the principal goal in the treatment of Type II diabetic patients for decades. The impact of pharmacological beta-cell stimulation on insulin pulsatility has not been examined in Type II diabetes, although the nature of the insulin response could be important to shortterm and long-term glucose control. In non-diabetic 
subjects, pharmacological beta-cell stimulation and inhibition occurs through modulation of insulin secretory burst mass with no changes of burst frequency [6-10].

Glucagon-like peptide-1 (GLP-1) is a peptide hormone that is secreted from the L cells of the intestinal mucosa in response to ingestion of mixed meals and glucose [11]. Glucagon-like peptide-1 potently stimulates insulin secretion and acts as an incretin hormone, potentiating glucose-stimulated insulin release. It also inhibits glucagon secretion and hepatic glucose output and as a consequence blood glucose declines [12].

Several mechanisms of humoral and neuronal origin have been proposed for generating the coordinated release pattern of insulin [13-15]. Glucagon-like peptide-1 itself has been shown to be secreted in a pulsatile manner during both basal and glucose-stimulated conditions, but the nature of its modulation of insulin pulsatility is not known [16]. Glucagon-like peptide- 1 infusion is able to re-establish beta-cell function as measured by first-phase and second-phase insulin response to a glucose challenge [17]. Analogously, GLP-1 infusion increases beta-cell sensitivity to minor ultradian glucose excursions under entrainment conditions in IGT but not in overt Type II diabetes [18].

The aim of our study was to determine the mechanistic effect of short-term GLP-1 infusion during glucose-clamped conditions on pulsatile insulin release as quantified by deconvolution analysis and on system regularity as assessed by a variety of time series analyses in Type II diabetic patients.

\section{Subjects and methods}

Subjects and design. The protocol was carried out in accordance with the Helsinki Declaration and was approved by the local ethical committee of Aarhus and Vejle Counties. Type II diabetic subjects $(n=8$, age $52 \pm 2$ years, BMI $29.4 \pm 0.9 \mathrm{~kg} /$ $\mathrm{m}^{2}$, fasting blood glucose $10.9 \pm 0.8 \mathrm{mmol}^{\prime}, \mathrm{HbA}_{1 \mathrm{c}} 7.3 \pm 0.6$ ) were recruited from the outpatient clinics of Medical Department, Kolding Sygehus and Department of Endocrinology and Metabolism, Aarhus University Hospital. Among the eight subjects studied, one was treated with metformin, three with sulphonylurea and three with a combination of the two. The study subjects received no antidiabetic medication for $48 \mathrm{~h}$ before the study and took no other medications known to influence beta-cell function or insulin sensitivity. The subjects were asked to maintain their usual diet and habits of physical exercise for 3 days before investigations.

Protocol. Synthetic human GLP-1 (7-36)amide was purchased from Peninsula Europe (Merseyside, St. Helens, UK). The peptide was dissolved in $0.9 \%$ saline containing $1 \%$ human serum albumin (Albumin Nordisk, Novo Nordisk, Bagsvaerd, Denmark; guaranteed to be free of hepatiti-B surface antigen and human immunodeficiency virus antibodies) and subjected to sterile filtration. Appropriate amounts of peptide for each experimental subject were dispensed into glass ampoules and stored frozen under sterile conditions until the day of the experiment.
The effect of GLP-1 on pulsatile insulin secretion was studied in an unblinded, placebo-controlled, randomised crossover design. Subjects were studied on two occasions with no less than 2 weeks intervening. Studies were carried out after a 10 -h overnight fast. At 0800 hours subjects were placed in a bed and intravenous catheters were inserted in each antecubital vein for sampling and infusion purposes. After $30 \mathrm{~min}$ of rest $(t=0)$, blood was collected every minute for 75 min (basal period). The patients were thereafter infused with either GLP-1 $\left(1.2 \mathrm{pmol} \cdot \mathrm{kg}^{-1} \cdot \mathrm{min}^{-1}\right)$ dissolved in isotonic saline containing $1 \%$ human albumin or saline with human albumin only until the end of the study. After $30 \mathrm{~min}$ of infusion, i.e. from 105 to $180 \mathrm{~min}$, blood was sampled every minute for $75 \mathrm{~min}$ (stimulated period). Throughout the study, plasma glucose was measured every 5-15 min and a variable infusion of $20 \%$ glucose was given to maintain constant glycaemia. The infusion rate was increased only in minor steps $\left(<0.7 \mathrm{mg} \cdot \mathrm{kg}^{-1} \cdot \mathrm{min}^{-1}\right)$ and was never decreased to avoid any glucose infusion-induced changes in insulin pulsatility. Further details on the sampling procedure have been previously described [9]. Additional blood samples were collected every $15 \mathrm{~min}$ for measurement of $\mathrm{C}$ peptide and GLP-1. Blood samples were stored at $-20^{\circ} \mathrm{C}$ and analysed within a month.

Assays. All biochemical analyses were performed in duplicate. Serum insulin concentration was measured using a two-site immunospecific ELISA [19]. The detection range was 3-600 $\mathrm{pmol} \cdot \mathrm{l}^{-1}$. At medium $\left(150 \mathrm{pmol} \cdot \mathrm{l}^{-1}\right)$, medium-high (200 $\left.\mathrm{pmol} \cdot \mathrm{l}^{-1}\right)$ and high $\left(350 \mathrm{pmol} \cdot \mathrm{l}^{-1}\right)$ concentrations, the inter-assay coefficients of variation were $4.5,4.9$ and $5.5 \%$, respectively and the intra-assay coefficients of variation were $2.8,2.6$ and $2.4 \%$, respectively. The antibodies cross-react 30 and $63 \%$ with 65,66 split proinsulin and des- 64,65 proinsulin, respectively, with no cross-reactivity with proinsulin, 32,33 split proinsulin and des- 31,32 proinsulin, C peptide, IGF-I, IGF-II and glucagon. Plasma glucose concentrations were measured by the glucose oxidation method (Beckman Instruments, Palo Alto, Calif., USA). Measurements of C peptide were done by a two-site monoclonal based ELISA assay (K6218, Dako Diagnostics, Cambridgeshire, UK). This assay has an intra-assay and interassay variation coefficient (for triplicates) of $2.2 \%$ and $3.3 \%$, respectively. Finally plasma GLP-1 was measured against standards of synthetic GLP-1 (proglucagon 78-107amide) of intestinal origin, using an antiserum highly specific for the amidated Cterminus of GLP-1. It measures the sum of intact GLP-1 and the primary metabolite, GLP-1 (9-39amide), which is formed in the circulation. The measured concentration therefore accurately reflects the amount of GLP-1 that was infused. The assay has a lower detection limit of $1 \mathrm{pmol} / \mathrm{l}$ and an intra-assay coefficient of variation below $5 \%$ at $20 \mathrm{pmol} / \mathrm{l}$.

Deconvolution analysis. Serum insulin concentration time series were analysed in a blinded manner by deconvolution analysis to quantify basal secretion, interpulse interval, secretory burst mass and amplitude [20]. Deconvolution was carried out with a previously validated iterative multiparameter technique, using the following assumptions: (1) the hormone is secreted in a finite number of bursts each with (2) an individual amplitude and (3) a common half-duration. Bursts are superimposed on a basal time-invariant secretory rate and (4) insulin is removed according to a bi-exponential disappearance rate as described previously [21]. The half-lives of insulin were assumed to be 2.8 and 5 min with a fractional slow compartment of $28 \%$ [21]. The fraction of total insulin released in a pulsatile fashion was calculated as:

Secretory burst mass/interpulse interval $\overline{\text { Secretory burst mass/interpulse interval + basal secretion }}$ 
Approximate entropy. Regularity of insulin concentration time series was assessed by the model-independent and scale-invariant statistic approximate entropy (ApEn) [22]. Approximate entropy measures the logarithmic likelihood that runs of patterns that are close (within $r$ ) for $\mathrm{m}$ contiguous observations remain close (within the tolerance width $r$ ) on subsequent incremental comparisons. A precise mathematical definition is given elsewhere [22]. Approximate entropy is considered a family of parameters dependent on the choice of the input parameters $m$ and $r$ and is to be compared only when applied to time series of equal length, as we do here. In this study ApEn was calculated with $r=0.2 \times \mathrm{SD}$ in the individual time series and $m=1$. To obviate the effect of trends in the time series ApEn was calculated on the first difference of data. A larger absolute value of ApEn indicates a higher degree of process randomness. Approximate entropy is rather stable to noise that lies within the tolerance width $r$. This is a standard way of doing ApEn in non-stationary time series.

Spectral analysis. To eliminate non-stationarity, spectral analysis was done on residuals after subtraction of a fitted line calculated as a seven-point centred equal-weighted moving average. Spectral analysis was done using a non-commercial software based on a previously described procedure [23]. Different window lengths were tested and a balance between stability and fidelity was obtained by using a Tukey window of 25 data points and the spectra were normalised using the assumption that the total variance in each time series was $100 \%$. This enables comparison of spectral estimates despite the different absolute values of insulin. The dominant peaks during basal and stimulated conditions were compared statistically.

Autocorrelation analysis. Autocorrelation analysis was done using the statistical software package SPSS version 9.0. The analysis was carried out on the unsmoothed data in which non-stationarity had been eliminated as described for spectral analysis. The correlation coefficients of the first non-negative peak of the autocorrelogram were compared statistically [24].

Statistical analysis. Statistical comparisons were done using SPSS version 9.0. Data were compared by ANOVA adjusting for treatment, sequence and subject. A significance level of 0.05 was used.

\section{Results}

Concentrations of hormones and plasma glucose. The infusion of GLP-1 resulted in an immediate rise of the GLP-1 concentration, stabilising at a mean of approximately $70 \mathrm{pmol} / \mathrm{l}$. Serum insulin and C peptide increased approximately $100 \%$ and there was a slight upward trend during the stimulation period. The mean plasma glucose was clamped at the concentration of the basal period. Mean circulating concentrations of C peptide, plasma glucose and GLP-1 along with the glucose infusion rate needed to maintain glycaemia are shown in Figure 1. One representative example of insulin concentration profiles under basal and stimulated conditions is shown in Figure 2, where increased absolute values and larger amplitudes are seen during GLP-1 infusion.
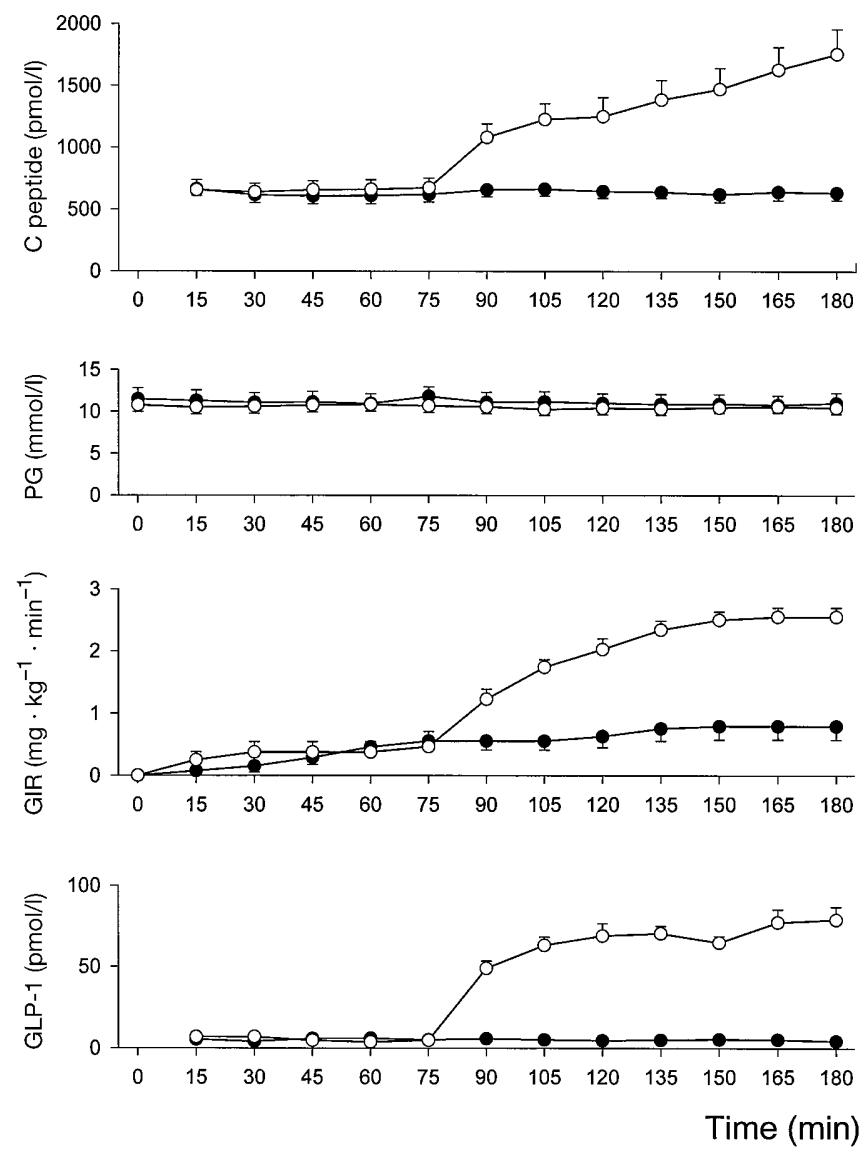

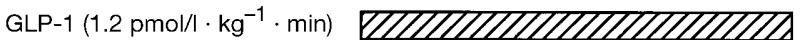

Fig. 1. Mean values for C-peptide concentration, plasma glucose concentration, glucose infusion rate and GLP-1 concentration during the basal and the stimulated period at the day of GLP-1 (O) and saline $(\mathcal{O})$ infusion. Data are means \pm SEM. The GLP-1 level is at near steady state during the stimulation period, while C-peptide concentration is slightly increasing. Plasma glucose is clamped at a constant concentration by variable glucose infusion

Deconvolution analysis. The principal results of deconvolution analysis are listed in Table 1 . The overall increase in insulin secretion was achieved by a parallel rise in basal and pulsatile insulin secretion. The fraction of insulin secreted in pulses was about $50 \%$ and remained unchanged during GLP-1 infusion. One example of a deconvolved insulin time series at baseline and during GLP-1 infusion is shown (Figure 3). This figure illustrates the increase in secretory burst mass and burst amplitude with no change in burst frequency.

Regularity statistics. Approximate entropy was applied to first difference derivatives of insulin concentration time series. At baseline there was no correlation between ApEn and fasting plasma glucose. Approximate entropy did not change $(p>0.5)$ after GLP-1 infusion compared with saline infusion, indicating an unchanged system regularity (Table 1 ). 

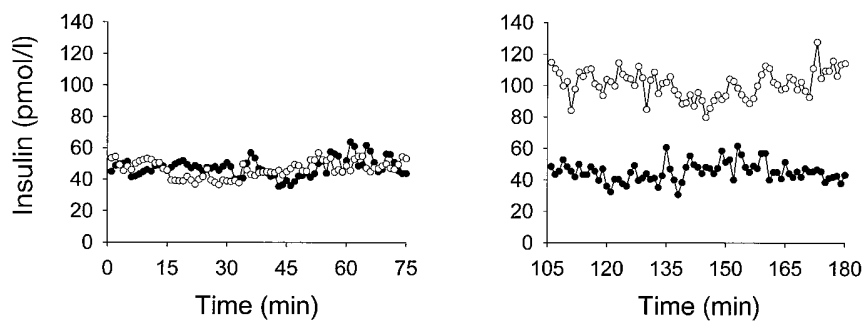

Fig. 2. One representative example of insulin concentration profiles during the basal period and the stimulated period at the day of GLP-1 $(\bigcirc)$ and saline $(O)$ infusion

Spectral analysis and autocorrelation analysis. The power and periodicity of the dominant spectral peak were determined by normalised spectral analysis. No statistically significant changes in spectral power were observed after GLP-1 infusion compared with saline. The interpulse interval was about $6 \mathrm{~min} /$ pulse and was unchanged by GLP-1 infusion. The frequency estimates obtained by spectral analysis and deconvolution analysis were similar. Spectral power and frequency estimates are listed in Table 1. Regular oscillatory activity was also evaluated by autocorrelation analysis. No statistically significant change was observed in the maximum autocorrelation coefficient after GLP-1 infusion compared with saline (Table 1). There was no correlation between fasting plasma glucose and spectral power or autocorrelation coefficient at baseline.

\section{Discussion}

The mechanisms underlying disruption of high-frequency pulsatile insulin secretion in Type II diabetes is not known. Likewise, the possibility of re-establishing the intra-islet and inter-islet coordination of insu-
A
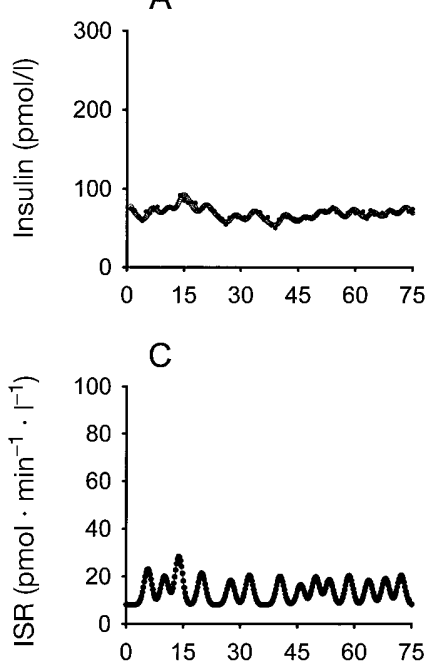

Time (min)
B

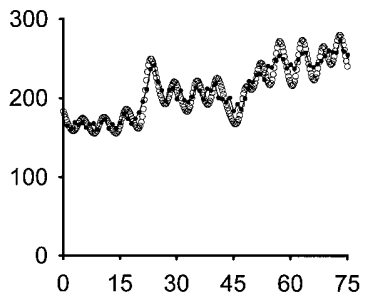

D

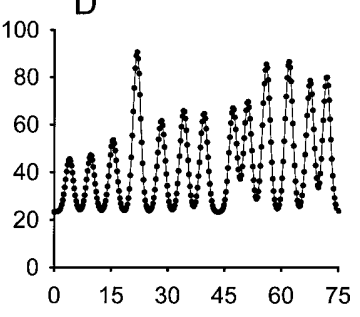

Time (min)

Fig.3. One representative example of deconvolution analysis of insulin concentration profiles. Panel A and B show concentration profiles (-O-) and best fit estimation $(\mathrm{O})$. Panel $\mathrm{C}$ and $\mathrm{D}$ show calculated insulin secretory rates (ISR). Panel A and $\mathrm{C}$ are during basal period and panel $\mathrm{B}$ and $\mathrm{D}$ are during GLP1 stimulation. Increased basal secretion and amplitude of pulsatile insulin secretion are seen during GLP-1 infusion

lin secretion is undefined. Glucagon-like peptide1 has been shown to re-establish first-phase and second-phase insulin secretion and beta-cell response to arginine in Type II diabetes subjects to values not different from those in control subjects [17]. Furthermore, in IGT subjects, GLP-1 infusion has been reported to enhance beta-cell sensitivity to glucose oscillations in an ultradian glucose-entrainment protocol [18]. It seems, thus, that GLP-1 exerts actions not only on the quantity of insulin expelled but also on

Table 1. Analysis of insulin concentration time series during basal and stimulated conditions at the day of saline and GLP-1 infusion

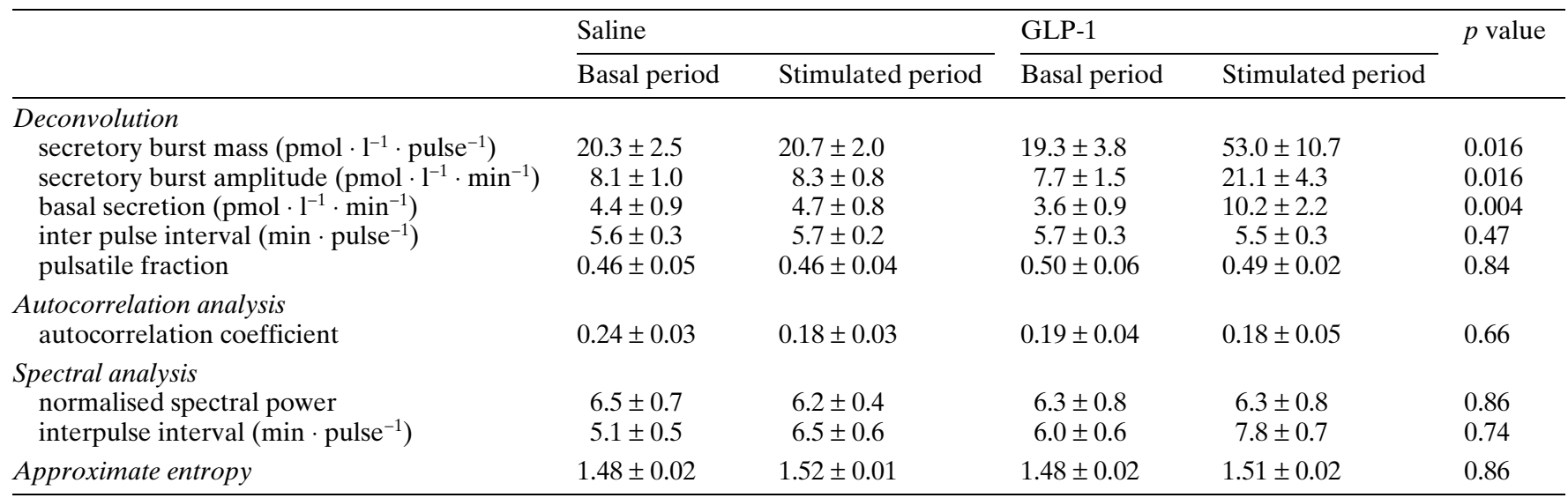

Data are given as means \pm SEM. $p$ values are calculated by ANOVA adjusting for treatment, sequence and subject. $p$ values for secretory burst mass and secretory burst amplitude are corrected using Bonferroni's correction 
the beta-cell coordination during short-term induced hyperglycaemia or an oscillatory exogenous supply of glucose. This peptide could, therefore, also prove capable of improving rapid pulsatile insulin secretion that depends on coordinated mobilisation of immediately releasable insulin stores.

The stimulatory effect of GLP-1 in diabetic patients might arise mechanistically by an increase in pulsatile insulin release (increasing burst mass or burst frequency) or by increased basal secretion. In healthy control subjects, GLP-1 infusion stimulates insulin secretion by increasing pulsatile as well as basal secretion with no change in burst frequency [9]. An improved regularity of release pattern was not expected in this study of healthy subjects. In Type II diabetes GLP-1 has, in a preliminary report, also been shown to increase insulin secretion by amplification of the burst mass with no effect on the burst frequency when GLP-1 was given as an overnight infusion [25]. This is similar to the findings in our study. In addition the absolute values of insulin secretory burst mass in the two studies are similar.

In this study, we evaluated the short-term action of GLP-1 on pulsatile insulin secretion in Type II diabetes with application of additional time series analyses. We have recently shown that ApEn is able to uncover changes in regularity dynamics following short-term beta-cell exposure to another secretagogue, repaglinide [10]. No restoration, nor any additional deterioration of system regularity in response to GLP-1 infusion is observed in the present data when evaluated by the same statistic. Additional time series analyses also disclosed no trend towards altered regularity or coordination, indicating that this finding is independent of the mathematical approach applied. Different results might have been obtained following long-term infusion of GLP-1 but short-term stimulation as carried out in this study would seem to best resemble the postprandial situation with a rather steep increase of GLP-1 concentration to a maximum of 50-60 $\mathrm{pmol} / \mathrm{l}[26]$.

The approximate $100 \%$ increase in overall insulin concentration is achieved by an increase in secretory burst mass and amplitude, with no effect on interpulse interval. This seems to be a general feature in the modulation of insulin secretion [6-10]. A preserved or enhanced amplitude of insulin secretion could be important for the peripheral actions of insulin [3-5]. The constant frequency could be a consequence of a feedback system involving a fixed circulation time, enzymatic processes which are unaffected by the different compounds given, or an intrapancreatic neuronal pacemaker with a constant periodicity.

The GLP-1 effect on stimulus-secretion coupling involves several distinctive processes. It inhibits $\mathrm{K}^{+}-$ ATP channel activity, resulting in a membrane depolarisation, increases the intracellular $\mathrm{Ca}^{2+}$-concentration by opening voltage-dependent $\mathrm{Ca}^{2+}$ channels and promotes $\mathrm{Ca}^{2+}$-induced insulin secretion by more distal and independent mechanisms [27]. The fact that GLP-1 infusion is not able to improve system regularity despite actions on various levels of the beta-cell sensing and secretion could imply that the defect involved in the deterioration of beta-cell coordination is not so readily reversible at this stage of diabetes. This notion would be in accordance with the finding that GLP-1 infusion improves stimulussecretion coupling during glucose entrainment in IGT subjects but not in overt diabetes [18]. In the entrainment study, the GLP-1 effect seemed to be a direct action of the secretagogue rather than a consequence of a decline in blood glucose as these subjects had only marginally increased plasma glucose concentrations. The glucose-clamped conditions in the present study are therefore unlikely to mask a potential GLP-1 effect.

We report that enhanced insulin secretion after GLP-1 infusion in diabetic patients is achieved by amplification of insulin secretory burst mass with no effect on burst frequency or beta-cell coordination and orderliness. That no improvement in coordination can be achieved in these diabetic patients together with previous findings might imply that early intervention is necessary to prevent this facet of beta-cell defect in Type II diabetes.

Acknowledgements. We thank A. Mengel and E. Horneman for excellent technical assistance. The study was supported by the Danish Research Council, Foundation for Medical Research, Vejle County, the Danish Diabetes Association, The Dandy Foundation, Vejle and Novo Nordisk, Bagsværd.

\section{References}

1. Polonsky KS, Given BD, Hirsch LJ et al. (1988) Abnormal patterns of insulin secretion in non-insulin-dependent diabetes mellitus. N Engl J Med 318: 1231-1239

2. Lang DA, Matthews DR, Burnett M, Turner RC (1981) Brief, irregular oscillations of basal plasma insulin and glucose concentrations in diabetic man. Diabetes 30: 435-439

3. Matthews DR, Naylor BA, Jones RG, Ward GM, Turner RC (1983) Pulsatile insulin has greater hypoglycemic effect than continuous delivery. Diabetes 32: 617-621

4. Komjati M, Bratusch-Marrain P, Waldhausl W (1986) Superior efficacy of pulsatile versus continuous hormone exposure on hepatic glucose production in vitro. Endocrinology 118: 312-319

5. Bratusch-Marrain PR, Komjati M, Waldhausl WK (1986) Efficacy of pulsatile versus continuous insulin administration on hepatic glucose production and glucose utilization in type I diabetic humans. Diabetes 35: 922-926

6. Porksen N, Hussain MA, Bianda TL et al. (1997) IGF-I inhibits burst mass of pulsatile insulin secretion at supraphysiological and low IGF-I infusion rates. Am J Physiol 272: E352-E358

7. Porksen N, Munn SR, Steers JL, Veldhuis JD, Butler PC (1996) Effects of somatostatin on pulsatile insulin secretion: elective inhibition of insulin burst mass. Am J Physiol 270: E1043-E1049 
8. Matthews DR, Lang DA, Burnett MA, Turner RC (1983) Control of pulsatile insulin secretion in man. Diabetologia 24: 231-237

9. Porksen NK, Grøfte T, Nyholm B et al. (1998) Glucagonlike peptide 1 increases mass but not frequency or orderliness of pulsatile insulin secretion. Diabetes 47: 45-49

10. Juhl CB, Porksen NK, Sturis J et al. (1999) Repaglinide amplifies insulin secretory burst mass without affecting burst frequency. Diabetes [Suppl] A245 (Abstract)

11. Orskov C (1992) Glucagon-like peptide-1, a new hormone of the entero-insular axis. Diabetologia 35: 701-711

12. Komatsu R, Matsuyama T, Namba M et al. (1989) Glucagonostatic and insulinotropic action of glucagonlike peptide I-(7-36)-amide. Diabetes 38: 902-905

13. Porksen N, Munn S, Ferguson D, O'Brien T, Veldhuis J, Butler P (1994) Coordinate pulsatile insulin secretion by chronic intraportally transplanted islets in the isolated perfused rat liver. J Clin Invest 94: 219-227

14. Jonas JC, Gilon P, Henquin JC (1998) Temporal and quantitative correlations between insulin secretion and stably elevated or oscillatory cytoplasmic $\mathrm{Ca}^{2+}$ in mouse pancreatic beta-cells. Diabetes 47: 1266-1273

15. Meneilly GS, Veldhuis JD, Elahi D (1999) Disruption of the pulsatile and entropic modes of insulin release during an unvarying glucose stimulus in elderly individuals. J Clin Endocrinol Metab 84: 1938-1943

16. Balks HJ, Holst JJ, von-zur-Muhlen A, Brabant G (1997) Rapid oscillations in plasma glucagon-like peptide-1 (GLP-1) in humans: cholinergic control of GLP-1 secretion via muscarinic receptors. J Clin Endocrinol Metab 82: 786-790

17. Rachman J, Gribble FM, Barrow BA, Levy JC, Buchanan KD, Turner RC (1996) Normalization of insulin responses to glucose by overnight infusion of glucagon-like peptide 1 (7-36) amide in patients with NIDDM. Diabetes 45: $1524-1530$
18. Byrne MM, Gliem K, Wank U et al. (1998) Glucagon-like peptide 1 improves the ability of the beta-cell to sense and respond to glucose in subjects with impaired glucose tolerance. Diabetes 47: 1259-1265

19. Andersen L, Dinesen B, Jorgensen PN, Poulsen F, Roder ME (1993) Enzyme immunoassay for intact human insulin in serum or plasma. Clin Chem 39: $578-582$

20. Veldhuis JD, Carlson ML, Johnson ML (1987) The pituitary gland secretes in bursts: appraising the nature of glandular secretory impulses by simultaneous multiple-parameter deconvolution of plasma hormone concentrations. Proc Natl Acad Sci USA 84: 7686-7690

21. Porksen NK, Nyholm B, Veldhuis JD, Butler PC, Schmitz O (1997) In humans at least $75 \%$ of insulin secretion arises from punctuated secretory bursts. Am J Physiol 273: E908-E914

22. Pincus SM (1991) Approximate Entropy as a measure of system complexity. Proc Natl Acad Sci USA 88: 2297-2301

23. Jenkins GM, Watts DG (1966) Spectral analysis and its applications. Holden-Day, San Francisco

24. Chatfield C (1996) The analysis of time series. An introduction. Chapman and Hall, London

25. Ritzel R, Schulte M, Nauck M et al. (1998) GLP-1 increases secretory burst mass of pulsatile insulin secretion in patients with impaired glucose tolerance and Type II diabetes. Diabetologia [Suppl 1] A182 (Abstract)

26. Orskov C, Wettergren A, Holst JJ (1996) Secretion of the incretin hormones glucagon-like peptide- 1 and gastric inhibitory polypeptide correlates with insulin secretion in normal man throughout the day. Scand J Gastroenterol 31: 665-670

27. Gromada J, Bokvist K, Ding WG, Holst JJ, Nielsen JH, Rorsman P (1998) Glucagon-like peptide 1 (7-36) amide stimulates exocytosis in human pancreatic beta-cells by both proximal and distal regulatory steps in stimulus-secretion coupling. Diabetes 47: 57-65 